\title{
The effectiveness and safety of acupuncture for the treatment of myasthenia gravis: a systematic review and meta-analysis of randomized controlled trials
}

\author{
Xue Zhang ${ }^{1,2}$, Wentao Ding ${ }^{2}$, Zhao Wang ${ }^{2}$, Xiuli Gu ${ }^{2}$, Wenzeng Zhu ${ }^{1}$ \\ ${ }^{1}$ China Academy of Chinese Medical Sciences, Guang'anmen Hospital, Beijing 100053, China; ${ }^{2}$ China Academy of Chinese Medical Sciences, South \\ Area of Guang'anmen Hospital, Beijing 102618, China \\ Contributions: (I) Conception and design: X Zhang, W Zhu; (II) Administrative support: W Zhu; (III) Provision of study materials or patients: X Zhu, \\ W Ding; (IV) Collection and assembly of data: X Zhu, W Ding; (V) Data analysis and interpretation: X Gu, Z Wang; (VI) Manuscript writing: All \\ authors; (VII) Final approval of manuscript: All authors. \\ Correspondence to: Wenzeng Zhu. China Academy of Chinese Medical Sciences, Guang'anmen Hospital, No. 5 Beixiange Street, Xicheng District, \\ Beijing 100053, China. Email: zhuwenzeng530@163.com.
}

Background: No systematic reviews of acupuncture as a treatment for myasthenia gravis (MG) have been published in English. The aim of our study is to evaluate the efficacy and safety of acupuncture as a treatment for MG.

Methods: We searched for randomized controlled trials (RCTs) in seven main electronic databases. Unpublished articles, including conference papers and Chinese doctoral and master's theses, were also included as supplementary sources. The primary outcome was the relative clinical score (RCS) response rate. We performed a meta-analysis using RR and MD with 95\% CI.

Results: Thirteen RCTs involving a total of 775 participants were included. Most included trials had a high risk of bias in allocation concealment and blinding. Eleven RCTs used acupuncture as an adjuvant to medication, and this treatment showed a significant improvement in the RCS response rate compared to medication alone (RR: 1.42; 95\% CI: 1.06-1.91; $\mathrm{P}=0.02$ ). The subgroup analysis based on the treatment duration showed a significant effect on the RCS response rate when the treatment duration was longer than 12 weeks (RR: 2.02; 95\% CI: 1.31-3.12; P=0.001). In contrast, there was no significant effect of treatment with a duration less than 8 weeks (RR: 1.14; 95\% CI: 0.91-1.44; P=0.26). Four RCTs showed a significant difference in the absolute clinical score (ACS) (RR: 3.42; 95\% CI: 1.23-5.61; P=0.002). The acupuncture group reported better outcomes. No severe adverse events corresponding to acupuncture were reported.

Conclusions: This meta-analysis suggests that acupuncture as an integrative therapy has a significant positive effect in treating MG. Acupuncture may enhance the efficacy of medication in MG patients. The safety of acupuncture requires further investigation. The clinical significance of these changes needs to be investigated by further studies using rigorous designs and longer follow-up times.

Keywords: Review; meta-analysis; myasthenia gravis (MG); acupuncture; relative clinical score (RCS)

Submitted Mar 27, 2019. Accepted for publication Sep 10, 2019.

doi: 10.21037/apm.2019.10.10

View this article at: http://dx.doi.org/10.21037/apm.2019.10.10

\section{Introduction}

Myasthenia gravis (MG) is an autoimmune disease in which autoantibodies interfere with neuromuscular transmission. The characteristics of MG include fluctuating muscle weakness and fatigability in various muscle groups, often resulting in obvious impairment in activities of daily living (ADL) $(1,2)$. The clinical course of this disease ranges from remission during an early stage to acute exacerbation and 
potentially even death (3). MG reportedly has a worldwide prevalence of 40-180 cases per million people and an annual incidence of $4-12$ cases per million people $(4,5)$. More recent estimates of the prevalence and incidence of MG tend to be higher than older estimate (6). Previously, long-term drug treatment was necessary for nearly all patients with MG. These treatments include cholinesterase inhibitors, immunosuppressants, plasma exchange, and thymectomy (7). However, these often-costly treatments are unable to completely cure MG, and most MG patients are refractory to drug treatment and prone to severe side effects, such as diarrhoea, nausea, vomiting, salivating, muscle twitching, delayed onset of action, and strong dependence. Additionally, most drug treatments are unable to inhibit the relapse of symptoms and achieve complete remission in MG patients (8-10).

Acupuncture is a significant component of complementary and alternative medicine and is widely used in clinical practice for the treatment of many diseases in China (11). Acupuncture is often used to treat MG but seldom used alone, which is usually used in combination with drugs. The acupuncture treatment of MG is considered to provide a synergistic effect and enhance the efficacy of drugs.

Thus, whether acupuncture can effectively treat MG is still a controversial issue. Acupuncture refers to a group of therapeutic techniques characterized by the insertion of needles into the skin, followed by either manual manipulation or electrical stimulation. Multiple theories have been proposed to explain the mechanisms of action, but the most widely investigated theory is that acupuncture treatment for MG increases acetylcholine receptor (AChR) expression at the neuromuscular junction (9). Regrettably, RCTs investigating acupuncture for the treatment of MG did not use AChR antibodies as an outcome index, which may be due to the previous difficulty in detecting AChR antibodies. Therefore, people pay more attention to improving the symptoms of MG by acupuncture. To date, no systematic reviews or meta-analyses of acupuncture for the treatment of MG have been reported in English. Our systematic review evaluated the efficacy and safety of acupuncture as a treatment for MG, offers suggestions for future research, and presents additional treatment options for such patients.

\section{Methods}

\section{Protocol and registration}

The systematic review reported here was performed in accordance with the Preferred Reporting Items for Systematic Reviews and Meta-Analyses (PRISMA) guidelines (12). The protocol of this systematic review and meta-analysis was registered in PROSPERO (13).

\section{Eligibility criteria}

Our study includes only randomized controlled trials (RCTs) investigating acupuncture for the treatment of $\mathrm{MG}$, and no language restrictions were applied. The participants were patients diagnosed with MG. There were no limitations regarding age, sex, or race. The intervention comparisons consisted of acupuncture compared with no treatment/ placebo/sham acupuncture/other active therapies or acupuncture in addition to another active therapy compared with the same active therapy alone. The following types of studies were excluded: (I) trials investigating acupuncture only compared with another form of acupuncture or selections of different acupuncture points compared with control groups and (II) studies involving different medicines in the experimental or control groups.

\section{Outcomes}

The primary outcome was the relative clinical score (RCS) response rate, which is the indicator most widely used to evaluate MG treatment in China (14). The RCS is an evaluation scale invented by a Chinese physician named Hanhao $\mathrm{Xu}$ that is similar to the MG score. This scale can sensitively respond to improvements in MG symptoms. The RCS response rate was defined as $50 \%$ or greater improvement of the RCS, representing significant improvement in symptoms, and was regarded as a dichotomous measure (effective or ineffective). The secondary outcomes included the absolute clinical score (ACS), quality of life and adverse effects. We conducted a comprehensive descriptive analysis of the adverse effects in the included and excluded clinical studies.

\section{Information sources and search}

The search strategy was based on the Cochrane Handbook guidelines (15). The search terms addressed the following three features: disease, intervention and study type. The following seven electronic databases were searched for studies published on or before 31 July 2018: PubMed, the Cochrane Central Register of Controlled Trials (CENTRAL), Embase, the Chinese Biomedical Literature 
Database (CBM), Wanfang Data, VIP and the China National Knowledge Infrastructure (CNKI). Unpublished articles, including conference papers and Chinese doctoral and master's theses, were also searched for as supplementary sources in CNKI. The reviewers obtained and screened the full texts of all other articles for eligibility.

\section{Study selection}

Two authors (XZ and WD) independently screened the studies and assessed the eligibility of the articles. All search results were input into EndNote X7 software (Thomson Reuters, New York, NY, USA) by the reviewers, and duplicate documents were deleted. If the two raters disagreed, the issue was resolved by discussion. The final decision was made by a third rater (WZ) if the disagreement was not resolved. The selection process was documented in a PRISMA flow chart (12).

\section{Data collection process and data items}

The data collection and analysis were conducted in the following steps: data extraction, quality assessment of the data, addressing the missing data, assessment of heterogeneity, subgroup analysis, sensitivity analysis, and grading of the quality of the evidence. Two authors independently screened the studies and extracted the data. The authors' names, publication year, methods, interventions, sample size, outcomes, adverse events, and related information were extracted from each study using a predesigned data extraction form. In the case of missing data, the original investigators were contacted by phone or e-mail.

\section{Quality assessment}

The methodological quality of the included trials was evaluated by two independent authors ( $\mathrm{ZW}$ and $\mathrm{XG}$ ) using the Cochrane Risk of Bias Tool (16). Disagreements regarding study eligibility were resolved by discussion, a third review author (WZ), or rechecking the source papers as necessary. The "risk of bias" assessments were categorized into the following three levels: low risk, high risk, and unclear risk.

\section{Data synthesis and statistical analysis}

The use of different types of acupuncture in the included studies may induce heterogeneity in the results.
Consequently, the data were pooled using a random-effects model. Heterogeneity was measured using both Cochran's $\mathrm{Q}$-test ( $\mathrm{P}$ value $\leq 0.10$ was used to define a significant degree of heterogeneity) and the $\mathrm{I}^{2}$ statistic $\left(\mathrm{I}^{2}>50 \%\right.$ showed the existence of heterogeneity) (16). A subgroup analysis was conducted to analyse the primary outcome based on the treatment duration. Sensitivity analyses were performed on the basis of the particular types of acupuncture.

The statistical analyses were conducted using RevMan 5.3 (International Business Machines Corporation, China) software, which was used to generate forest plots of the pooled relative risks of the primary and secondary outcomes with $95 \%$ confidence intervals (CIs). Since the primary outcome was a dichotomous variable, we combined the risk ratios (RRs) of all studies and calculated the 95\% CI. Except for the adverse events, the secondary outcomes were continuous data; therefore, we combined the mean differences (MDs) of all studies and calculated the 95\% CI. The publication bias was explored via a funnel-plot analysis.

\section{Results}

\section{Study selection}

Figure 1 shows the search and selection process. Using an electronic literature search, we identified 598 references; of these references, we excluded 552 articles after screening the titles and abstracts. Forty-six references were fully reviewed, and 13 RCTs (17-29) involving a total of 775 screened participants were selected for inclusion in our qualitative and quantitative analyses. Two of the 13 RCTs were unpublished theses for a master's degree $(25,26)$.

\section{Study characteristics}

All included trials were performed in China and published in Chinese between 2007 and 2017. All included studies reported similar baseline characteristics. Regarding the type of acupuncture, 2 studies $(17,18)$. investigated the effect of warming electroacupuncture (EA), which is performed using the following procedure: moxibustion strips are placed on the handles of certain needles, and electric stimulators are attached to the other needles. Six studies (20-25) investigated the effect of manual acupuncture alone, two studies investigated the effect of warming acupuncture $(19,28), 1$ study $(28)$ investigated the effect of warming acupuncture plus moxibustion and acupressure, 1 study (28) investigated the effect of acupuncture plus message, 


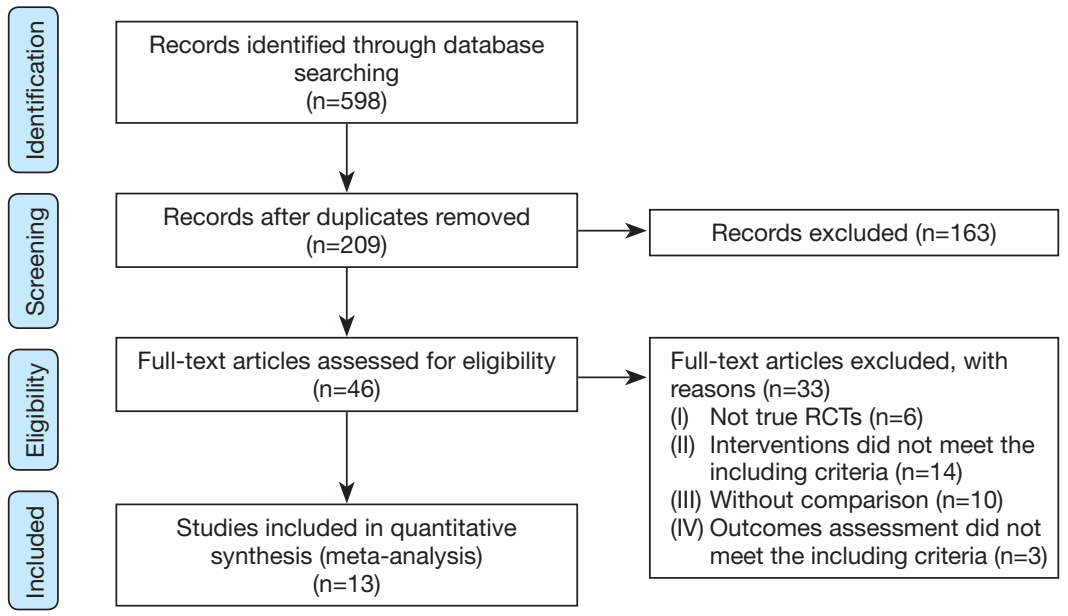

Figure 1 Flowchart of trial selection process for this systematic review.

and 1 study (29) investigated the effect of acupuncture plus moxibustion. The sample sizes ranged from 30 to 82 , and the treatment duration varied from 4 weeks to 6 months. None of the trials used sham acupuncture as a control. All included Western medication regimens were immunosuppressants, steroids, or their combination. One RCT (19) mentioned a follow-up period of 3 months; none of the other trials mentioned any follow-up. The details of the included trials are listed in Table 1.

Nine of the 13 trials reported the RCS response rate. Only four trials reported the ACS. The RCS response rate was defined as $50 \%$ or greater improvement in the RCS, representing significant improvement in symptoms. The RCS is calculated as follows: RCS = (baseline ACS-ACS after treatment)/baseline ACS ${ }^{*} 100 \%$ (30). The method used to calculate the ACS incorporates the changes in muscle strength and the degree of muscle fatigue. We combined all outcomes by category. Then, new data were extracted as dichotomous outcomes for the RCS response rate and continuous outcomes for the ACS. We also examined whether safety was assessed in the included trials.

\section{Risk of bias}

Four studies $(19,23,25,26)$ conducted randomization with random number tables, and three studies $(17,18,20)$ used a lottery system. Six studies simply mentioned randomization but did not specify the method. The processes of concealed allocation and blinding in the included trials were not clearly described and could not be ascertained, even after contacting the authors for more information. The performance bias and blinding of all trials were judged as "unclear" due to the lack of blinding of the participants between the intervention groups. The blinding of the outcome assessors in all trials was judged as "high" risk of bias because the reports of the studies did not include this precaution. Four RCTs reported the RCS response rate without providing the ACS. Thus, we concluded that there was a risk of incomplete outcome data among these four trials. Only one trial (21) reported the number of dropouts, and three trials reported the number of adverse events. Therefore, the other nine RCTs were judged as having a "high" risk of selective outcome reporting risk. The overall quality of the trials was low, and most trials were evaluated as having an "unclear" or "high risk of bias" (Figure 2). The funnel plots of these RCTs show no publication bias (Figure 3).

\section{Meta-analysis results}

Thirteen RCTs were divided into 2 categories for a metaanalysis based on the different types of intervention groups.

\section{Acupuncture plus medication versus medication}

\section{Relative clinical score response rate}

Eight trials involving a total of 476 participants examined acupuncture as an adjunct to medication. The combined result of the meta-analysis showed that the combination of acupuncture and medication was more effective than the same medication alone in improving the RCS response rate (RR: $1.42 ; 95 \% \mathrm{CI}: 1.06-1.91 ; \mathrm{P}=0.02$ ) with high 


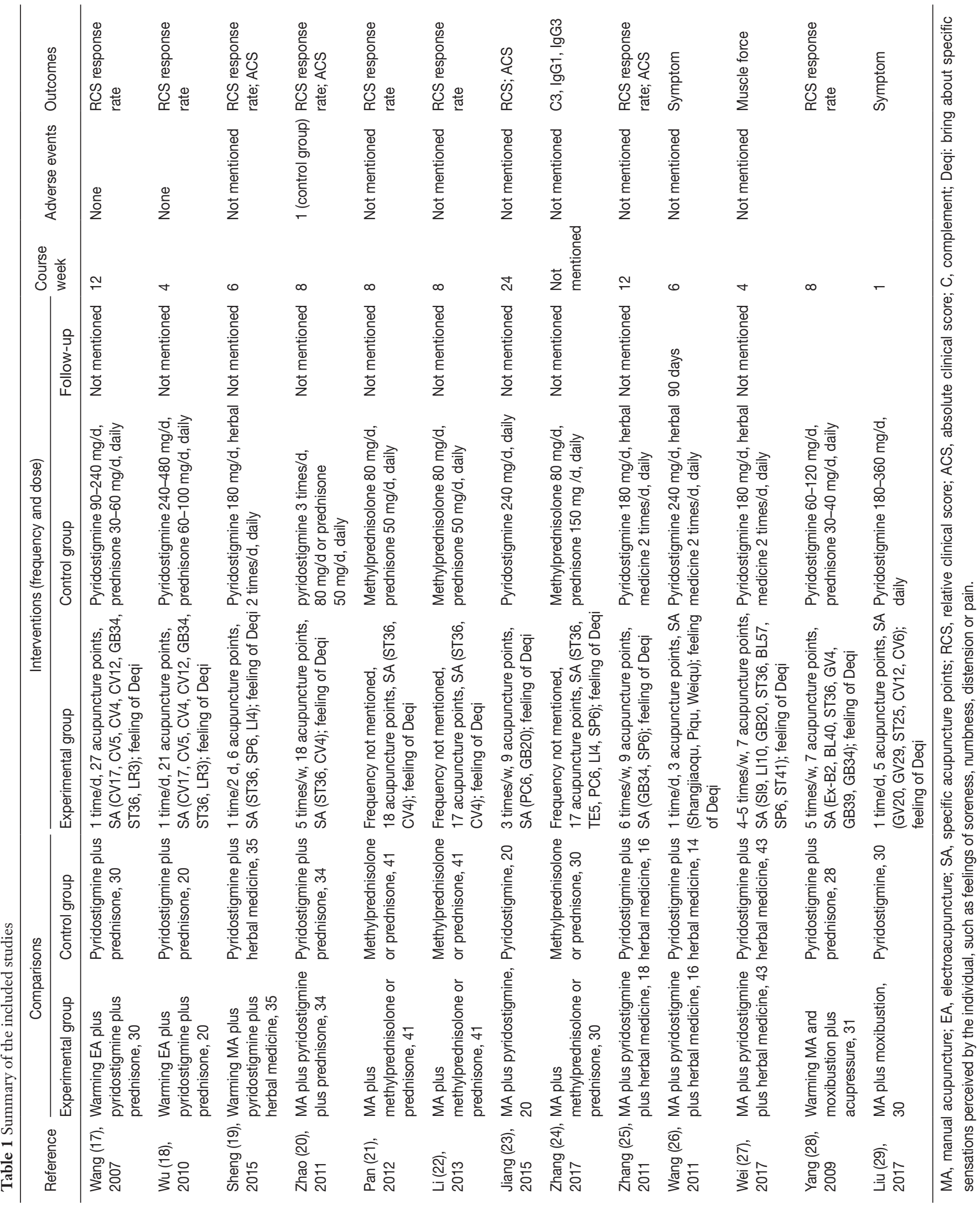




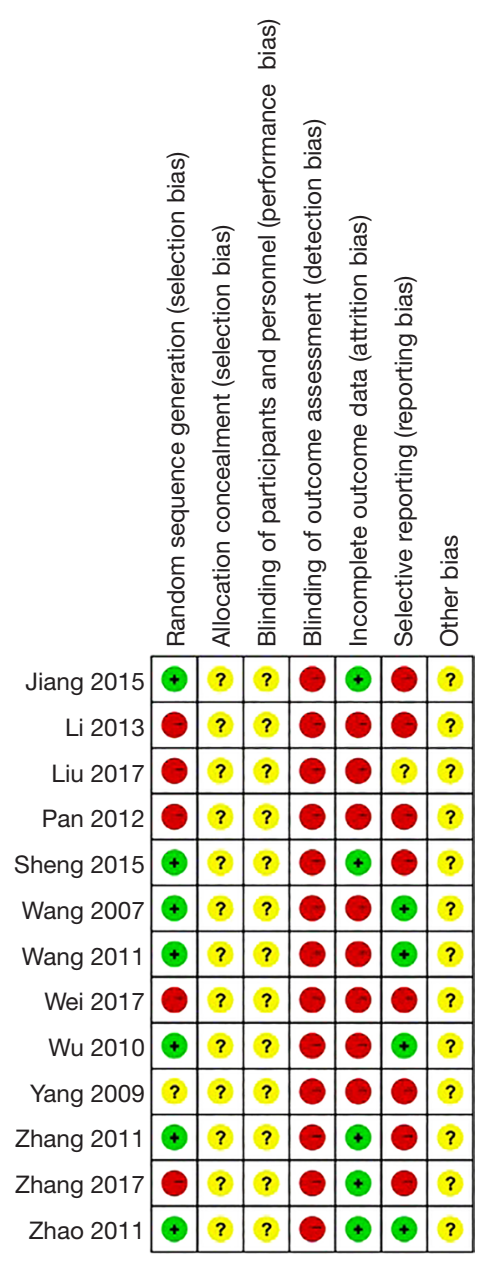

Figure 2 Risk of bias summary of included studies.

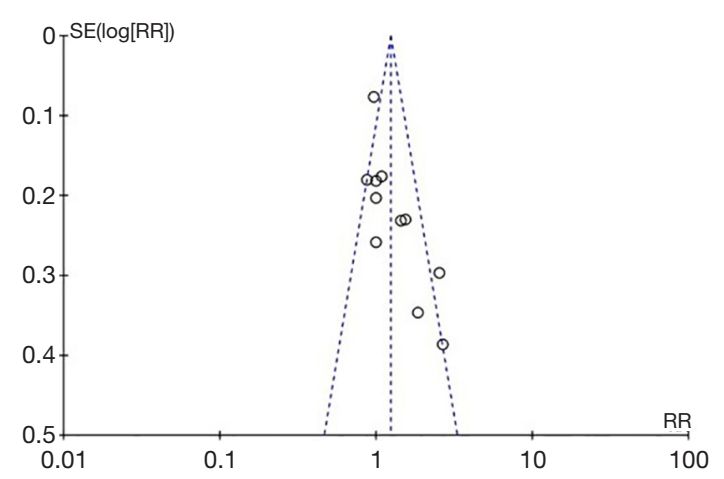

Figure 3 The funnel plots.

heterogeneity $\left(\mathrm{I}^{2}=76 \%\right)$ (Figure 4).

A subgroup analysis was performed based on the different treatment durations. The treatment duration in three RCTs was longer than 12 weeks, and that in the remaining five
RCTs was less than 8 weeks. The meta-analysis results of the treatments lasting 12 weeks in duration showed a statistically significant difference between the two groups (RR: $2.02 ; 95 \%$ CI: $1.31-3.12 ; \mathrm{P}=0.001)$ with moderate heterogeneity $\left(\mathrm{I}^{2}=40 \%\right)$ (Figure 3$)$. The other meta-analysis of the treatments lasting less than 8 weeks showed no statistically significant difference between the two groups (RR: $1.14 ; 95 \%$ CI: 0.91-1.44; $\mathrm{P}=0.26)$ with moderate heterogeneity $\left(\mathrm{I}^{2}=53 \%\right)$ (Figure 4$)$.

We performed sensitivity analyses based on four particular types of acupuncture (warming EA and warming acupuncture) while pooling the data from these thirteen trials. None of these analyses changed the statistical significance of the RCS response rate with particular types of acupuncture compared with drugs, and there was no heterogeneity among the studies in any analyses.

\section{ACS}

Four RCTs involving 212 participants evaluated the change in ACS. The meta-analysis of the data showed that the combination of acupuncture and medication had a better effect in improving ACS than medication alone (RR: 3.42; 95\% CI: 1.23-5.61; $\mathrm{P}=0.002$ ) (Figure 5).

\section{Quality of life}

Only a single study involving 40 participants evaluated the quality of life. The SF-36 Questionnaire was used to investigate the quality of life of MG patients. The results of the study showed that the use of acupuncture as an adjunct resulted in a greater effect in improving the quality of life.

\section{Acupuncture versus medication}

Relative clinical score response rate: one $\mathrm{RCT}^{29}$ involving a total of 59 participants compared warming acupuncture and moxibustion with pyridostigmine plus prednisone. The results showed that compared to Western medicine, the combination of acupuncture and moxibustion had a greater effect in improving the RCS response rate (RR: 1.67; $95 \%$ CI: $1.07-2.59 ; \mathrm{P}=0.02$ ).

\section{Adverse events}

We included all clinical trials investigating acupuncture as a treatment for MG in the safety evaluation. Only 3 of the 50 clinical articles reported the occurrence of adverse events. One case of respiratory muscle paralysis occurred in a control group treated with medication alone (17). One trial (31) reported 2 cases of slight dry mouth and nausea, but these symptoms disappeared after 2 weeks. One trial (32) 


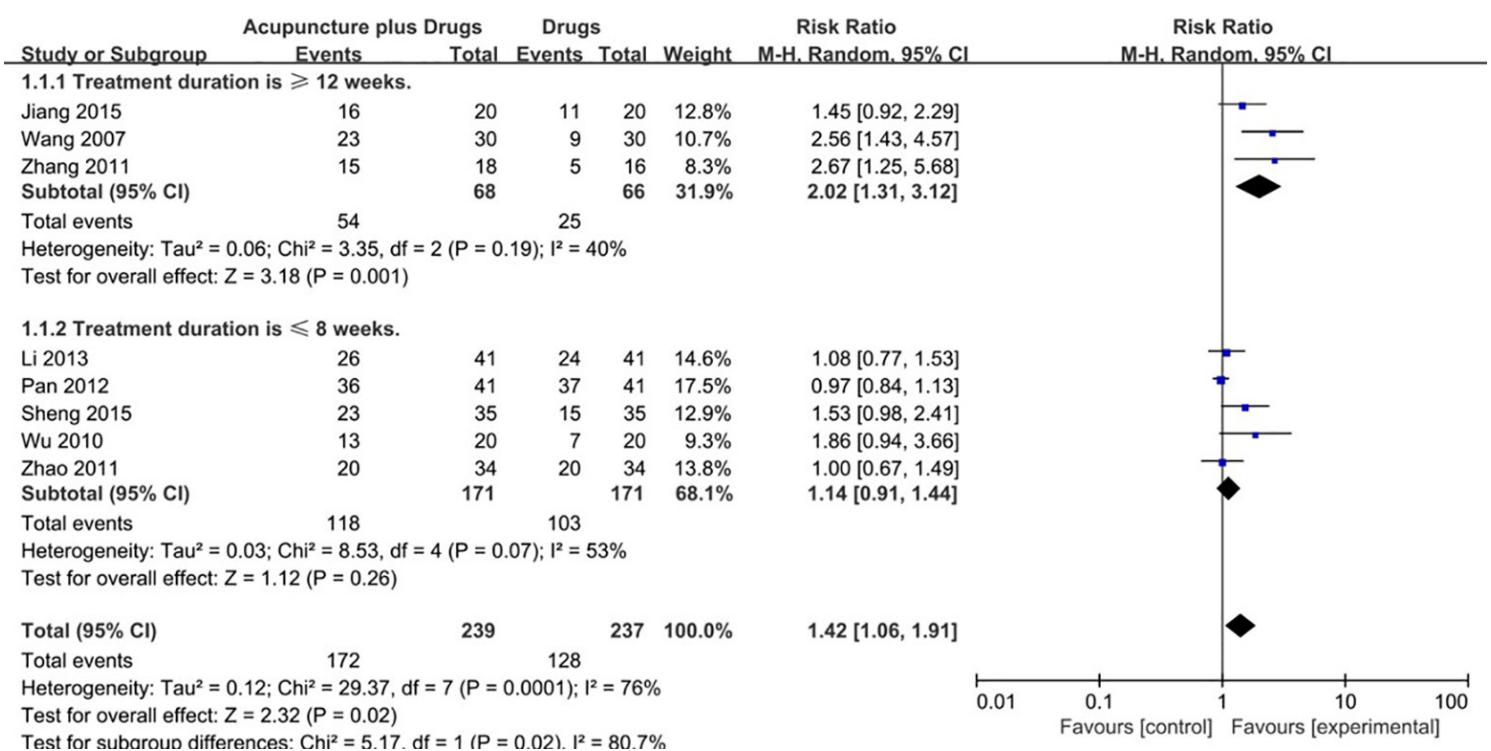

Figure 4 Forest plot of comparison: acupuncture plus drugs versus drugs; outcome: RCS. RCS, relative clinical score.

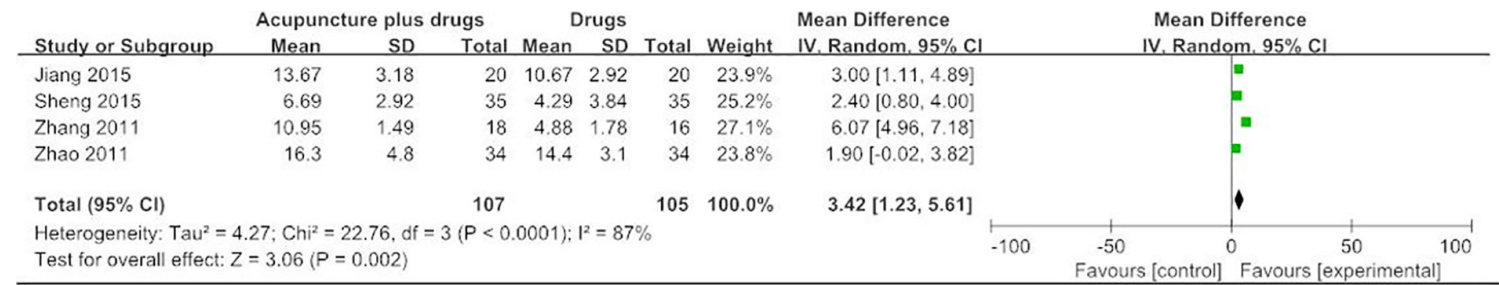

Figure 5 Forest plot of comparison: acupuncture plus drugs versus drugs; outcome: ACS. ACS, absolute clinical score.

reported 3 cases of myasthenic crisis in the acupuncture group and 6 cases of myasthenic crisis in the control group. The remaining studies did not report any adverse reactions or events related to acupuncture.

\section{Discussion}

To the best of our knowledge, this study presents the first meta-analysis exploring acupuncture as a treatment for MG. This systematic review summarizes the available evidence from RCTs regarding the efficacy and safety of acupuncture as a treatment for MG. In this review, we included RCTs comparing acupuncture or acupuncture combined with medication to medication alone; we did not include any trials comparing acupuncture to sham acupuncture or no treatment due to the lack of related studies. Thirteen RCTs meeting our inclusion criteria were included. Although the reported data were obtained from low-quality trials, the results showed that long-term acupuncture therapies (longer than 12 weeks) may be beneficial in improving RCS. Considering the insufficient number of studies, further research is needed to explore the use of acupuncture as a treatment for MG. In this study, we analysed the patients' RCS response rate to compare the different degrees of improvement across different studies. In the subgroup analysis, the results showed that the effect of treatment with a duration longer than 12 weeks was superior to that of treatment with a duration less than 8 weeks. This result seemed to indicate that a longer treatment duration could enhance the therapeutic effect. The sensitivity analysis showed that there was no heterogeneity based on two particular types of acupuncture (warming acupuncture and warming EA), indicating that the effectiveness of acupuncture against MG is likely not affected by the type of acupuncture. Altogether, long-term acupuncture treatment may be beneficial for MG treatment. However, the inadequate number of included RCTs, the lack of placebo-controlled trials, the insufficient sample size, and 
the different study durations may also explain these results. Further studies are needed to explore the reasons for the heterogeneity of the effects.

In this meta-analysis, we also assessed the differences between acupuncture alone and acupuncture along with medication in improving the ACS and quality of life. The combined results showed the specific therapeutic effect of acupuncture combined with medication in improving the ACS. However, the effect of acupuncture in the improvement of the quality of life was uncertain. Only 1 study measured this indicator. We conducted a descriptive analysis due to the variety of adverse reactions and different types of clinical studies included. To date, most studies have mainly focused on the effectiveness of acupuncture. Only 2 trials involving 5 cases from 50 clinical articles reported tolerable adverse reactions and deterioration of disease. Thus, the safety of acupuncture is uncertain due to the lack of attention to adverse events among the clinical trials.

The overall quality of the evidence in this metaanalysis as evaluated by the Grading of Recommendations Assessment, Development and Evaluation (GRADE) (33) approach was low. There was generally a high risk of bias in the 13 included trials. Moreover, all studies had methodological problems, such as unclear randomization and allocation concealment processes, small sample sizes, and the lack of assessor blinding. The significant heterogeneity might result from the different types of acupuncture. Previous publications have reported the longterm effects of acupuncture at follow-up visits (34). These studies showed that persistent effects existed for several months to years after the end of the treatment period. Nevertheless, only one of the thirteen trials included in this meta-analysis reported a follow-up period, which was fairly short at 3 months. Furthermore, the specific choice of Western medication and dose and acupuncture intervention methods varied, and both these factors decreased the reliability of the results.

Acupuncture may be beneficial to MG patients; however, the uncertainty of its curative effect, safety, persistence and acceptability in the treatment of MG has prevented its widespread use for the treatment of this condition. We expect that additional high-quality RCTs with placebocontrolled trials examining the use of acupuncture as a treatment for MG involving larger sample sizes, more outcomes and longer observation periods will be conducted to assess the clinical value and safety of acupuncture. Moreover, the adverse events should be identified and reported in future RCTs. Safety assessments should include physical examinations, electrocardiography, and vital sign checks during the study.

This meta-analysis has several limitations. First, due to the lack of reporting of placebo-controlled trials comparing penetrating acupuncture and nonpenetrating acupuncture, the placebo effect of acupuncture cannot be excluded. Clinical trials comparing acupuncture with sham acupuncture are needed in the future. Second, the ACh antibody is an important indicator used to evaluate the efficacy of MG, but it is not used in RCTs related to acupuncture. Third, a case report showed that acupuncture could reduce the recurrence of MG (35), but none of the studies in the present analysis reported the recurrence rate because the follow-up periods were short or absent. Fourth, the studies did not include deterioration rates or full safety assessments. Future clinical studies should include deterioration rates as an outcome index. Fifth, although the effect of acupuncture on MG may depend on the severity of the disease, there was barely any mention of this consideration in the included trials. Therefore, the results of the current analysis need to be interpreted with caution.

\section{Conclusions}

This meta-analysis suggests that an integrative therapy involving both acupuncture and medication may be beneficial for patients with MG but only with longterm therapy. Acupuncture may enhance the efficacy of medication in MG patients. The safety of acupuncture also requires further investigation. The clinical significance of these findings needs to be examined in further studies adopting rigorous designs and longer follow-up times.

The search strategies used in PubMed were as follows:

(I) To identify relevant outcomes (myasthenia gravis): "Myasthenia gravis" [Mesh] OR asthenic bulbar paralysis [Title/Abstract] OR muscular dystrophy [Title/Abstract] OR myasthenia [Title/Abstract]) OR muscular weakness [Title/Abstract] OR chronic weakness [Title/Abstract];

(II) To identify relevant exposures (acupuncture): Acupuncture therapy $[\mathrm{MeSH}]$ OR Acupuncture [Title/Abstract] OR Acupuncture points [Title/ Abstract] OR Acupuncture* [Title/Abstract] OR Body acupuncture [Title/Abstract] OR Scalp acupuncture [Title/Abstract] OR Manual acupuncture [Title/Abstract] OR Auricular acupuncture [Title/Abstract] OR Ear acupuncture [Title/Abstract] OR Electroacupuncture [Title/ 
Abstract] OR Fire needling [Title/Abstract] OR Dermal needle [Title/Abstract] OR Plum blossom needle [Title/Abstract] OR Pyonex [Title/ Abstract] OR Abdominal acupuncture [Title/ Abstract] OR Filiform steel needle [Title/Abstract] OR Acupressure [Title/Abstract] OR Electric stimulation [Title/Abstract];

(III) To limit to epidemiologic studies (RCTs): Randomized controlled trial [Publication Type] OR Controlled clinical trial [Publication Type] OR Randomized [Title/Abstract] OR Randomly [Title/ Abstract] OR Placebo [Title/Abstract] OR Trial [Title/Abstract] OR Groups [Title/Abstract].

\section{Acknowledgments}

All authors are sincerely grateful to American Journal Experts for the editorial work.

\section{Footnote}

Conflicts of Interest: The authors have no conflicts of interest to declare.

Ethical Statement: The authors are accountable for all aspects of the work in ensuring that questions related to the accuracy or integrity of any part of the work are appropriately investigated and resolved.

\section{References}

1. Vrinten C, Lipka AF, van Zwet EW, et al. Ephedrine as add-on therapy for patients with myasthenia gravis: protocol for a series of randomised, placebo-controlled n-of-1 trials. BMJ Open 2015;5:e007863.

2. Skeie GO, Apostolski S, Evoli A, et al. Guidelines for treatment of autoimmune neuromuscular transmission disorders. Eur J Neurol 2010;17:893-902.

3. Owe JF, Daltveit AK, Gilhus NE. Causes of death among patients with myasthenia gravis in Norway between 1951 and 2001. J Neurol Neurosurg Psychiatry 2006;77:203-7.

4. Carr AS, Cardwell CR, McCarron PO, et al. A systematic review of population based epidemiological studies in Myasthenia Gravis. BMC Neurol 2010;10:46.

5. Heldal AT, Owe JF, Gilhus NE, et al. Seropositive myasthenia gravis: a nationwide epidemiologic study. Neurology 2009;73:150-1.

6. Gilhus NE, Verschuuren JJ. Myasthenia gravis: subgroup classification and therapeutic strategies. Lancet Neurol 2015;14:1023-36.

7. Mantegazza R, Bonanno S, Camera G, et al. Current and emerging therapies for the treatment of myasthenia gravis. Neuropsychiatr Dis Treat 2011;7:151-60.

8. Anderson D, Phan C, Johnston WS, et al. Rituximab in refractory myasthenia gravis: a prospective, open-label study with long-term follow-up. Ann Clin Transl Neurol 2016;3:552-5.

9. Huang HP, Pan H, Wang HF. "Warming yang and invigorating qi" acupuncture alters acetylcholine receptor expression in the neuromuscular junction of rats with experimental autoimmune myasthenia gravis. Neural Regen Res 2016;11:465-8.

10. Iorio R, Damato V, Alboini PE, et al. Efficacy and safety of rituximab for myasthenia gravis: a systematic review and meta-analysis. J Neurol 2015;262:1115-9.

11. Yang L, Cheng Y. Experimental autoimmune myasthenia gravis in rabbit induced by peptides T $\alpha 125-147$ (Chinese). Chinese Journey of Neurology 2003;36:32.

12. Liberati A, Altman DG, Tetzlaff J, et al. The PRISMA statement for reporting systematic reviews and metaanalyses of studies that evaluate health care interventions: explanation and elaboration. Ann Intern Med 2009;151:W65-94.

13. Xue Zhang. The effectiveness and safety of acupuncture and related therapies for myasthenia gravis: a systematic review and meta-analysis of randomized controlled trials. PROSPERO 2016:CRD42016046731. Available online: http://www.crd.york.ac.uk/PROSPERO/display_record. asp?ID=CRD42016046731

14. Xu XH. Diagnosis and treatment of myasthenia gravis (Chinese). New Chinese Medicine 2000;31:431-3.

15. Higgins JP, Thompson SG, Deeks JJ. Measuring inconsistency in meta-analyses. BMJ (Clinical research) 2003;327:557-60.

16. Green, JPTHaS. Cochrane Handbook for Systematic Reviews of Interventions, Version 5.3.0. The Cochrane Collaboration. 2011. Available online: http://handbook. cochrane.org/

17. Wang SH, Cui X, Feng J. Warming electroacupuncture combined with western medicine for treatment of myasthenia gravis and effect on IL-4 level in the patients. Zhongguo Zhen Jiu 2007;27:901-3.

18. Wu YT, Wang SH, Cui X. Effect of warm electroacupuncture on serum IL-6 in patients with myasthenia gravis (Chinese). Contemporary Medicine 2010;16:23-5. 
19. Sheng ZY, Chen G, Hu ZH. Clinical observation of traditional Chinese medicine in the treatment of myasthenia gravis with warming acupuncture (Chinese). Shanghai Journal of Acupuncture and Moxibustion 2015;34:540-2.

20. Zhao CC, Wang H, Shang JF. Effect of glucocorticoid and acupuncture on immunoglobulin and complement in patients with myasthenia gravis (Chinese). Chinese General Practice 2011;14:2897-9.

21. Pan XM. Effect of acupuncture combined with glucocorticoid on immunoglobulin and complement for patients with myasthenia gravis (Chinese). Chinese Cosmetic Medicine 2012;21:173-4.

22. Li WG. Effect of acupuncture combined with glucocorticoid on immunoglobulin and complement on patients with myasthenia gravis (Chinese). Journal of Practical Traditional Chinese Medicine 2013;19:331-2.

23. Jiang A, Li P. Clinical observation on the treatment of myasthenia gravis with Tong Du Tiao Shen acupuncture method (Chinese). Sichuan Traditional Chinese Medicine 2015;33:152-5.

24. Zhang YD. Effect of acupuncture combined with glucocorticoids on immunoglobulin and complement in patients with myasthenia gravis (Chinese). Chinese Practical Medicine 2017;12:105-7.

25. Zhang D. A clinical observation on acupuncture and medicine for myasthenia gravis of type IIA. Hunan: Hunan University of Traditional Chinese Medicine, 2011.

26. Wang N. A clinical study on eye acupuncture combined with Chinese medicine in the treatment of ocular myopathy myasthenia gravis. Liaoning: Liaoning University of Traditional Chinese Medicine, 2011.

27. Wei ZY, Li GW, Pang S, et al. A clinical study on the treatment of myasthenia gravis by the combination of

Cite this article as: Zhang X, Ding W, Wang Z, Gu X, Zhu W. The effectiveness and safety of acupuncture for the treatment of myasthenia gravis: a systematic review and meta-analysis of randomized controlled trials. Ann Palliat Med 2019;8(5):576-585. doi: 10.21037/apm.2019.10.10 traditional Chinese and Western Medicine (Chinese). Chinese Community Doctors 2017;33:97-9.

28. Yang B, Jia CW, Yin KJ. Treatment on 31 cases myasthenia gravis patients by Warming acupuncture combined with acupressure (Chinese). Shaanxi traditional Chinese Medicine 2009;30:332-3.

29. Liu XJ, Xie Q. Clinical Study of Acupuncture and Moxibustion on Ocular myopathy Myasthenia Gravis by Professor Xie Qiang (Chinese). Guangming traditional Chinese Medicine 2017;32:2761-3.

30. Wang XY, Xu XH. absolutely and relatively clinical score used for patients with myasthenia gravis (Chinese). Chinese Journal of Neurology 1997;30:87-90.

31. Xu FQ. The observation of the curative effect on Zhongiling granule in treatment of ocular myasthenia gravis (Chinese). Liaoning Journal of traditional Chinese Medicine 2006;33:134-6.

32. Li HY. Clinical observation on the treatment of myasthenia gravis with acupuncture and moxibustion (Chinese). Chinese Clinical Practice Medicine 2010;4:207-8.

33. GRADE Working Group. GRADE Handbook for Grading Quality of Evidence and Strength of Recommendations Version 3.2, 2013. Available online: http://www. gradeworkinggroup.org

34. MacPherson H, Vertosick EA, Foster NE, et al. The persistence of the effects of acupuncture after a course of treatment: a meta-analysis of patients with chronic pain. Pain 2017;158:784-93.

35. Le Panse, Cizeron-Clairac RG, Cuvelier M, et al. Regulatory and pathogenicw mechanisms in human autoimmune myasthenia gravis. Ann N Y Acad Sci 2008;1132:135-42. 